\title{
ОБОБЩЕННЫЙ УЩЕРБ ПРОМЫШЛЕННЫХ ПРЕДПРИЯТИИ, ОБУСЛОВЛЕННЫЙ ОГРАНИЧЕНИЕМ ЭЛЕКТРОСНАБЖЕНИЯ
}

\author{
K. JAANIMAXGI, M. MOTUS. ELEKTRITARBIMISE PIIRAMISEST TINGITUD TOOSTUSETTE- \\ VOTETE OLDISTATUD KAHJUM \\ K. JAANIMĂGI, M. MOTUS. GENERALIZED DAMAGE OF THE INDUSTRIAL ENTERPRISES \\ CAUSED BY THE LIMITS OF THE POWER SUPPLY
}

\section{(Прёдставил И. Эпик)}

Определению ущерба, причиняемого ограничением электроснабжения, посвящено много работ энергетиков различных научных школ ${ }^{*}\left[{ }^{1,2}\right]$, однако в большинстве из них само понятие «ущерб» не определено, даются лишь способы оценки тех или иных последствий отключения потребителей. Ниже предлагается определение ущерба, приносимого потребителям предполагаемым снижением нагрузки за счет изменения режима электропотребления. Определение ущерба основывается на использовании практически доступных исходных данных. Предполагается, что о сниже́нии нагрузки потребители предварительно предупреждены и что ограничение нагрузки не выходит за рамки аварийной и технологической брони отдельных потребителей.

На основе экспертного анализа при снижении нагрузки энергосистемой для ее оптимального распределения по потребителям предлагается учитывать восемь критериев, четыре из которых (от третьего до шестого) рассмотрены в $\left[{ }^{3}\right]$.

1. Достижение минимального снижения конечнюго * суммарного чистого продукта по всем предприятиям при ограничении потребления электроэнергии (в основном ограничение предприятий с относительно «дешевой» конечной чистой продукцией; в рублях на 1 кВт·ч использованной электроэнергии).

2. Уменьшение ожидаемого ущерба предприятий («вторичный» ущерб $\left.\left[{ }^{1}\right]\right)$, для которых данное предприятие является поставщиком (в основном ограничение предприятий с низкими величинами удельных поставок продукции на 1 кВт - ч используемой электроэнергии).

7. Преимущественное ограничение предприятий, имеющих меньшие основные и оборотные фонды на 1 кВт - ч используемой электроэнергии.

8. Преимущественное ограничение предприятий, работающих с менее дорогостоящим сырьем.

Выбранным критериям соответствует предлагаемая ниже математическая модель распределения ограничений потребляемой мощности и электроэнергии по отраслям или предприятиям промышленности

$$
f_{1}=\sum_{i=1}^{N} c_{1 i}\left(Q_{i} \cdot T-x_{i}\right) \rightarrow \min
$$

* Под конечным чнстым продуктом подразумевается продукт, выходящий из сферы производства и не поступающий на другие предприятия в качестве оборудования, деталей и материалов. 


$$
\begin{aligned}
& f_{2}=\sum_{i=1}^{N} c_{2 i}\left(Q_{i} \cdot T-x_{i}\right) \rightarrow \min , \\
& f_{3}=\sum_{i=1}^{N} \frac{W_{i}^{\max }}{W_{i}^{\text {yct }}}\left(Q_{i} \cdot T-x_{i}\right) \rightarrow \text { min } \\
& f_{4}=\sum_{i=1}^{N} 1 / c_{3 i}\left(Q_{i} \cdot T-x_{i}\right) \rightarrow \min , \\
& f_{5}=\sum_{i=1}^{N} c_{5 i}\left(Q_{i} \cdot T-x_{i}\right) \rightarrow \min , \\
& f_{6}=\sum_{i=1}^{N} c_{6 i}\left(Q_{i} \cdot T-x_{i}\right) \rightarrow \min , \\
& f_{7}=\sum_{i=1}^{N} c_{7 i}\left(Q_{i} \cdot T-x_{i}\right) \rightarrow \min , \\
& f_{8}=\sum_{i=1}^{N} c_{8 i}\left(Q_{i} \cdot T-x_{i}\right) \rightarrow \min , \\
& \sum_{i=1}^{N} x_{i}=x \cdot T
\end{aligned}
$$

$\left(\begin{array}{c}\text { Аварийная } \\ \text { бронь }\end{array}\right)$

$$
\leqslant x_{i} \leqslant Q_{i} \cdot T, \quad x_{i} \geqslant 0,
$$

где $W_{i}^{\text {ует }}, W_{i}^{\max }$ - установленная и максимальная мощности $i$-й отрасли, $c_{1 i}$ - удельная конечная чистая продукция $i$-й отрасли за вычетом соответствующей заработной платы**, руб./кВт· ч, $c_{2 i}-$ удельная чистая продукция $i$-й отрасли, поставляемая в производственную сферу, за вычетом соответствующей составляющей заработной платы **, руб./кВт - ч, $c_{3 i}$ - электроэнерговооруженность труда, кВт * - ч/чел., $c_{5 i}, c_{6 i}$ - часы использования максимальной мощности в сутки и установленной в год $i$-й отраслью, $c_{7 i}$ - удельные значения величины основных и оборотных фондов на 1 кВт - ч используемой электроэнергии, $c_{8 i}$ - удельная стоимость используемого сырья на 1 кВт.ч используемой электроэнергии, $x_{i}, Q_{i}$ - выделяемая в результате оптимизации потребность (на 1 ч) в электроэнергии для $i$-й отрасли, $x-$ суммарное для всех отраслей количество выделяемой электроэнергии на 1 ч, $N$ количество отраслей, $T$ - период времени, на который вводятся ограничения ***.

Задача (1)-(10) является линейной задачей векторного программирования. Один из возможных методов ее решения основывается на построении области эффективных решений (Парето) с последующим выделением из нее оптимального компромиссного решения. Таким образом, алгоритм решения задачи (1)-(10) содержит IV этапа.

I. Сведение целевых функционалов (1)-(8) к безразмерным.

II. Нахождение вектора идеальных решений

$$
f_{\text {ид }}=\left(f_{1}^{\text {onт }}, f_{2}^{\text {опт }}, \ldots, f_{8}^{\text {опт }}\right),
$$

** Составляющая заработной платы 
где $f_{i}$ опт - оптимальное значение функционала $f_{i}$ на области $(9)-(10)$. III. Построение на основе методов имитационного моделирования [ $\left.{ }^{4}\right]$ области Парето задачи (1)-(10).

IV. Выбор из области Парето решения наименее удаленного от идеального.

Решение, полученное на IV этапе, представляет собой оптимальное компромиссное решение в отношении распределения ограничений потребляемой мощности и электроэнергии по отраслям и предприятиям промышленности.

Этому решению соответствует обобщенный функционал $\left[{ }^{5}\right]$ вида $\phi(\bar{x})=\sum_{i=1}^{8} \lambda_{i} f_{i}$. Набор $\lambda_{i}$ фиксируется за счет выбора оптимального компромиссного решения, обеспечивающего минимальное значение функционала $\phi(\bar{x})$; $\phi(\bar{x})$ и назовем обобщенным ущербом совокупности предприятий.

Для решения задачи (1)-(10) применимы также методы, основанные на теории матричных игр $\left[{ }^{6,7}\right]$, и другие процедуры и методы $\left[{ }^{5}\right]$.

Приведенный алгоритм реализован на ЕC 1052. Наряду с оптймальным компромиссным решением, обеспечивающим минимальное значение обобщенного ущерба ф, программа выдает и значения функционалов $f_{i}$ в нем, что позволяет осуществить человеко-машинный режим за счет опроса ЛПР (лицо принимающее решение) $\left[{ }^{6,8}\right]$ с дополнительным вводом ограничений типа $f_{i} \leqslant \hat{f}_{i}$, что может обеспечить учет неформализуемых факторов и связей.

Отметим, что определение удельного ущерба для каждого отдель- . ного предприятия в силу отсутствия полного набора соответствующих исходных данных представляется нам очень сложной задачей. Этим и обусловлено появление вышеприведенного подхода.

Настоящая модель позволяет рассматривать и ситуации отключения потребителей, причем в этом случае вместо отраслей рассматривается совокупность предприятий, питаюшихся с одного фидера. Количество таких совокупностей соответствует количеству фидеров. В этом случае к $f_{7}$ и $f_{8}$ добавляются составляющие, соответствующие порче и ремонту оборудования и порче сырья $\left(\hat{c}_{7}\right.$ и $\hat{c}_{8}-$ соответствующие показатели, добавляемые к $c_{7}$ и $c_{8}$ соответственно). Учитывая, что $\hat{c_{7}}$ и $\hat{c_{8}}$ могут быть заданы лишь в интервале своих возможных значений, задача (1)-(10) переходит в задачу оптимизации в условиях неопределенности, для решения которой нужно применять суперпозицию методов многоцелевой оптимизации $\left[{ }^{5}\right]$ и оптимизации в условиях неопределенности $\left[{ }^{9}\right]$. В этом случае условие $(10)$ заменяется неравенствами $x_{i} \leqslant$ $\leqslant Q_{i} \cdot T$ и совокупность условий вида $T \leqslant T_{1}, x_{i} \leqslant \hat{x}_{i} \Rightarrow c_{7}=c_{7}+\hat{c}_{7}$; $\hat{c}_{7} \in\left[\hat{c}_{7}{ }^{1}, \hat{c}_{7}{ }^{2}\right]$, где $\hat{x}_{i}-$ аварийная бронь, что незначительно усложняет соответствующие программы для ЭВМ.

На основе решения получаемой задачи выявляется совокупность фидеров, подлежащих отключению при снижении нагрузки на определенную величину. Решая исходную задачу и применяя метод наименьших квадратов, возможно построение зависимостей обобщенного ущерба от глубины ограничения (от выделяемого количества электроэнергии), а также построение соответствующих очередей отключения фидеров.

Аналогичный подход применим и для определения обобщенного ущерба, обусловленного ограничением топливоснабжения $\left[{ }^{10}\right]$. 
1. Методические вопросы исследования надежности больших систем энергетики. Иркутск, СО АН СССР, вып. 18, 1980.

2. Окороков В. Р. Управление электроэнергетическими системами. Л., Изд-во ЛГУ, 1976.

3. Мытус М. М., Нурсте Х. О., Соосаар С. О., Яанимяги К. Э. Методические вопросы исследования надежности больших систем энергетики. Иркутск, СО АН СССР, вып. 23, 1981, 131-136.

4. Focke, J. Math. Operationsforsch. Statist., 4, № 5, 365-369 (1973).

5. Дехтяренко В. А., Своятыцкий Д. А. Препринт 76-30 Ин-та кибернетики АН УССР, Киев, 1976.

6. Яанимяги К. Изв. АН ЭССР, Физ. Матем., 28, № 4, 363-364 (1979).

7. Юттлер X. Экономика и матем. методы, 111, вып. 3, 397-406 (1967).

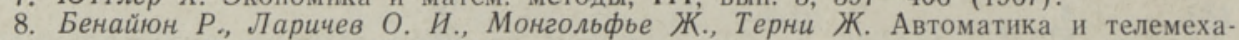
ника, № 8, 108-115 (1971).

9. Беляев Л. С. Решение сложных оптимизационных задач в условиях неопеделенности. Навосибирск, «Наука», 1978.

10. Мытус М., Яанимяги К. Изв. АН ЭССР, Физ. Матем., 30, № 1, $56-57$ (1981).

Ннститут термофизики и электрофизики Академии наук Эстонской ССР

Поступила в редакцию 21/III 1983 